J3eA, Journal sur l'enseignement des sciences et technologies de l'information et des systèmes, Volume 2, Hors-Série 2, 10 (2003)

DOI : http://dx.doi.org/10.1051/bib-j3ea:2003610

(C) EDP Sciences, 2003

\title{
Apport de la microthermie pour le refroidissement des systèmes
}

M. Ivanova*, Y. Avenas*, C. Schaeffer**, C. Gillot*** et A. Bricard****

* Laboratoire d'Électrotechnique de Grenoble ENSIEG

BP 46

F-38402 Saint Martin d'Hères Cedex, France

** CIME

46 avenue Félix Viallet

F-38000 Grenoble Cedex 4, France

*** CEA/LETI

17 rue des Martyrs

F-38054 Grenoble Cedex 9, France

**** CEA/GRETh

17 rue des Martyrs

F-38054 Grenoble Cedex 9, France 


\title{
Apport de la microthermie pour le refroidissement des systèmes
}

\author{
Mariya IVANOVA*, Yvan AVENAS*, Christian SCHAEFFER****, Charlotte GILLOT**, Alain \\ BRICARD***
}

\author{
*Laboratoire d'Electrotechnique de Grenoble ENSIEG - BP 46 - 38402 Saint Martin d'Hères cedex - France \\ CEA/LETI**/GRETh*** 17 rue des Martyrs - 38054 Grenoble cedex 9 - France \\ ****CIME 46 avenue Félix Viallet -38000 cedex 4 - France
}

Résumé - Aujourd'hui, on assiste simultanément à une augmentation des pertes thermiques au niveau des convertisseurs d'énergie et à une miniaturisation des systèmes électriques. Pour refroidir ces ensembles, on voit apparaître, depuis quelques années, des travaux utilisant des stratégies de microthermie pour évacuer les énergies dissipées et homogénéiser la température de ces systèmes. Dans cet article, nous aborderons le principe d'extraction par convection forcée puis nous présenterons un caloduc plat à rainures en silicium permettant d'épanouir les flux thermiques afin de mieux pouvoir les évacuer.

\section{Introduction}

Pour pénétrer de nouveaux domaines d'application, les systèmes électriques doivent se faire discrets. Dans ce contexte, leur volume et leur poids sont des critères devenus essentiels. Les convertisseurs de puissance ont été parmi les premiers systèmes électriques à suivre cette tendance. Cette évolution, rendue possible par l'augmentation des fréquences de fonctionnement des convertisseurs, s'est traduite par des niveaux de puissance dissipée et des densités de flux thermiques élevées pour l'ensemble des constituants des convertisseurs. Pour des composants semi-conducteurs tels que les IGBT, des densités de flux thermique de plus de $300 \mathrm{~W}$. $\mathrm{cm}^{-2} \mathrm{ont}^{\text {été }}$ générées. Dans ce contexte, le niveau d'efficacité des refroidisseurs associés à ces systèmes a dû être amđioré dans des proportions importantes. Malgré l'apport de la microthermie et les travaux conduits dans ce domaine, force est de constater que ce sont encore les performances thermiques du refroidisseur qui limitent le fonctionnement électrique des convert isseurs statiques. La gestion thermique d'un système électrique peut être assurée à partir du moment où le système de refroidissement assure l'extraction des énergies dissipées. Pour les applications considérées faiblement contraintes sur le plan thermique, la gestion thermique de l'équipement passe essentiellement par l'extraction des calories dissipées au sein du système électrique. Cette extraction est réalisée soit par convection forcée avec, par exemple, de l'air ou de l'eau, soit par un système passif faisant appel à un fonctionnement dans lequel le fluide a sa phase liquide en équilibre avec sa phase vapeur dans un milieu confiné. Pour les applications considérées plus fortement contraintes sur le plan thermique, une phase de répartition du flux thermique est nécessaire avant de réaliser l'extraction des calories [1].

\section{Fonctionnement d'un extracteur à fluide caloporteur à microcanaux :}

L'évolution des composants électroniques a conduit à une augmentation des densités de flux de chaleur dissipées, rendant insuffisant le refroidissement par air, dispositif le plus simple et le plus utilisé. La convection forcée d'un liquide permet d'améliorer considérablement les performances thermiques des systèmes électroniques. Proposés au début des années quatre-vingt pour le refroidissement des composants de la microélectronique comme les processeurs, les échangeurs à microcanaux s'avèrent particulièrement performants. Compacts, ils peuvent, dans le cas de l'électronique de puissance, être intégrés directement dans le module, à la place de la semelle en cuivre. La résistance thermique du système est alors réduite par la suppression de l'interface boîtier / refroidisseur constituée de graisse thermique et l'augmentation de l'échange convectif entre le fluide et les parois du refroidisseur. L'utilisation de refroidisseurs à microcanaux pour les composants électroniques a été proposée en premier lieu par D.B. Tuckerman et R.F.W. Pease [2]. Ils ont analysé le régime laminaire établi dans des canaux de section rectangulaire réalisés dans du silicium. Dans les nombreuses études qui ont suivi, les différents auteurs ont modifié et amélioré la structure initiale. Phillips [3] a publié une étude bibliographique complète concernant les refroidisseurs à microcanaux. Il donne une analyse détaillée des écoulements en régime laminaire ou turbulent, établi ou en cours de développement. Il étudie aussi l'effet de la variation des paramètres géométriques sur la résistance thermique (rapport des largeurs de l'ailette et du canal, haut eur et rapport de forme du canal, épaisseur du substrat, longueur des canaux). Knight [4] propose une analyse des régimes laminaire et turbulent pour un refroidissement avec de l'air. Il montre que, dans certains cas, le régime turbulent donne de meilleures performances. Il donne une méthode d'optimisation du nombre d'ailettes et compare son analyse à des résultats expérimentaux. Weisberg [5] résout numériquement par la méthode des éléments finis le problème thermohydraulique pour un régime laminaire établi et pour différents rapports de forme du canal. Il propose une méthode d'optimisation utilisant les résultats de la résolution numérique. 
Copeland [6] présente un refroidisseur à microcanaux avec de multiples entrées - sorties. Il donne des modèles analytiques et numériques ainsi que des résultats expérimentaux. Au cours de sa thèse [7], L. Meysenc a étudié le refroidissement de composants de puissance à l'aide de microcanaux. Il s'agissait de dispositifs avec une seule puce, le composant étant directement brasé sur un bloc de cuivre dans lequel avaient été usinés les canaux. C. Gillot a étendu sa démarche à des modules multipuces [8] .

\subsection{Analyse unidirectionnelle}

Cette analyse est utilisée par la plupart des auteurs pour le dimensionnement des refroidisseurs à microcanaux. L'échangeur sur lequel est fixé le composant est représenté sur la figure ci-après. Le fluide circulant dans les canaux permet d'évacuer la chaleur dissipée par le composant. Les hypothèses utilisées conduisent à considérer que la chaleur est générée uniformément sur la face supérieure du refroidisseur, que la conduction de la chaleur

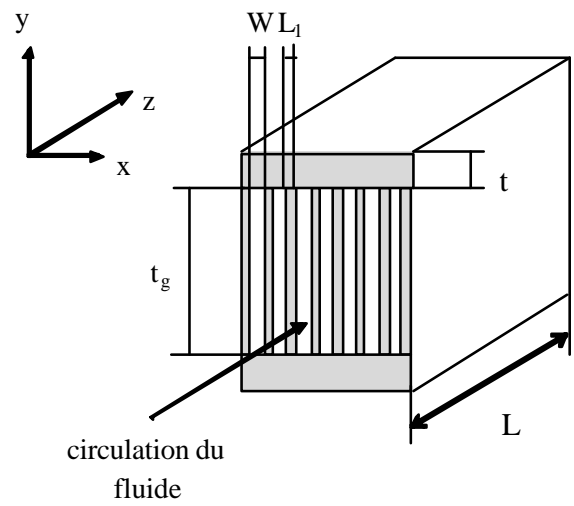

Figure 1: Principe d'un microrefroidisseur à microcanaux

dans le diffuseur du refroidisseur et dans les ailettes dans la direction de circulation du fluide est négligeable et que le flux de chaleur est uniforme sur les parois. Dans ces conditions, les profils de température du fluide et des parois du refroidisseur sont linéaires, et l'écart entre les deux est constant sur toute la longueur du refroidisseur. On peut alors définir deux résistances thermiques. La première, $R_{\text {thcap }}$, est due au réchauffement du fluide à travers les canaux. La seconde, $R_{\text {thconv }}$, modélise l'écart de température entre le fluide et la paroi des canaux, dû à la convection. Si l'on considère que la convection est caractérisée par un coefficient d'échange $h$ uniforme sur les parois et que la base inférieure des ailettes est adiabatique, un calcul analytique prenant en compte la conduction dans les ailettes donne $R_{\text {thconv }}$ :

$R_{\text {thconv }}=\frac{1}{h n L\left(2 \beta t_{g}+W\right)}$, où $\beta$ représente l'efficacité de l'ailette: $\beta=\frac{\tanh \left(\sqrt{2 h / L_{l} k_{p}} t_{g}\right)}{\sqrt{2 h / L_{l} k_{p} t_{g}}}$.

Ces formules permettent de définir la résistance thermique globale de l'échangeur par : $R_{t h}=R_{\text {thcap }}+R_{\text {thconv }}$

\subsection{Obtention du coefficient d'échange}

Le coefficient d'échange $h$ permet un e formulation globale de l'échange de chaleur entre le fluide et les parois du canal. Ce coefficient est fonction des propriétés du fluide, de la géométrie des canaux (forme, dimensions, rugosité des parois) et du type d'écoulement (laminaire, turbulent, đabli ou en cours de développement). Il est généralement obtenu par une analyse dimensionnelle faisant intervenir trois nombres.

1. Le nombre de Reynolds : il caractérise l'écoulement (laminaire ou turbulent). $\operatorname{Re}=\frac{? v D_{h}}{\mu}$,

2. Le nombre de Prandtl : il ne dépend que des propriétés physiques du fluide. $\operatorname{Pr}=\frac{\mu C_{p}}{k_{l}}$,

3. Le nombre de Nusselt : il donne la valeur du coefficient d'échange $h . N u=\frac{h D_{h}}{k_{l}}$,

avec $D_{h}$ diamètre hydraulique; qui pour un canal de section rectangulaire s'exprime par : $D_{h}=\frac{2 D l c}{(D+l c)}$. 
Le nombre de Nusselt est déterminé à l'aide de relations empiriques obtenues en corrélant des résultats issus de l'expérimentation avec un ensemble de nombres sans dimension. Dans la plupart des cas, le Nusselt s'exprime en fonction du Reynolds et du Prandtl. L'utilisation de ces corrélations nécessite la connaissance de leur domaine de validité. Différentes corrélations sont utilisées suivant le régime d'écoulement et la géométrie des canaux. Des tables permettent aussi de calculer le nombre de Nusselt pour des régimes non établis. Généralement établies pour des canaux de taille millimétrique, les corrélations doivent être vérifiées pour des canaux plus petits. Ainsi, par exemple, la limite entre les régimes laminaire et turbulent est souvent fixée, pour des canaux rectangulaires de taille millimétrique, à un Reynolds de l'ordre de 2300. D'après certains auteurs [9], cette limite intervient pour des Reynolds de 1000 à 1500 dans les canaux de petite taille (de quelques centaines de micromètres de large). Différentes corrélations sont rappelées dans la thèse de L. Meysenc [7]. Nous retiendrons à titre d'exemple celle qu'il a vérifiée pour des microcanaux de section rectangulaire usinés dans du cuivre, valable pour un régime laminaire établi, si le flux de chaleur est uniformément réparti sur trois faces des canaux, la face inférieure étant considérée adiabatique :

$N u=-14,859+65,623 G-71,907 G^{2}+29,384 G^{3}$. G est un paramètre géométrique introduit par A. Bejan [10]

tel que $G=\frac{\left(t_{g} / W\right)^{2}+1}{\left(t_{g} / W+1\right)^{2}}$. Pour les régimes non établi (hydrauliquement et/ou thermiquement), des tables permettent le calcul du Nusselt [11]. Citons une corrélation utilisable en régime turbulent :

$N u=0,012\left(\operatorname{Re}^{0,87}-280\right)+\operatorname{Pr}^{0,4}$ valable pour un Prandtl compris entre 1,5 et 500 et un Reynolds compris entre 3000 et $10^{6}$.

\subsection{Calcul des contraintes}

Pour prendre en compte les contraintes liées à l'écoulement du fluide, il reste à calculer les pertes de pression le long des canaux. Pour cela, on introduit un quatrième nombre sans dimension, appelé coefficient de frottement :

$f=\frac{t_{p}}{\frac{1}{2} ? v^{2}}$.En régime établi, les pertes de pression sont données par $? P=4 f \frac{L}{D_{h}} ? \frac{v^{2}}{2}$. Comme pour le nombre de Nusselt, le coefficient de frottement est obtenu par des corrélations en fonction du nombre de Reynolds. En régime laminaire établi [7], le coefficient de frottement peut être calculé par la formule :

$f=\frac{4,7+19,64 G}{\operatorname{Re}}$.

En régime turbulent, on peut utiliser la relation suivante : $f=\left(0,0929+1,01612 D_{h} / L\right) R e^{-0,268-0,3193 D_{h} / L}$. La puissance nécessaire à la circulation du fluide dans l'échangeur à microcanaux s'exprime par le produit des pertes de pression par le débit volumique total.

\subsection{Optimisation}

L'approche analytique ainsi que les corrélations donnant le coefficient d'échange décrites dans les paragraphes précédents permettent d'optimiser les performances des refroidisseurs à microcanaux. Le problème consiste à déterminer les grandeurs géométriques du canal et la vitesse du fluide permettant d'obtenir la résistance thermique la plus faible, tout en respectant les contraintes de l'écoulement que sont les pertes de pression totales ou la puissance motrice. Les grandeurs du problème sont classées en quatre groupes.

\begin{tabular}{|l|l|} 
LES DONNEES DU PROBLEME & $\begin{array}{l}\bullet \text { taille de la puce } \\
\bullet \text { propriétés thermiques du socle et de l'ensemble des constituants } \\
\bullet \text { type de fluide et ses propriétés }\end{array}$ \\
\hline $\begin{array}{l}\text { LES GRANDEURS A } \\
\text { OPTIMISER }\end{array}$ & $\begin{array}{l}\bullet \text { profondeur d'un canal } \\
\bullet \text { largeur d'un canal } \\
\bullet \text { largeur d'une ailette } \\
\bullet \text { vitesse du fluide }\end{array}$ \\
\hline LES CONTRAINTES & $\begin{array}{l}\bullet \text { pertes de pression totales } \\
\bullet \text { puissance motrice utile }\end{array}$ \\
\hline LA GRANDEUR A MINIMISER & $\bullet$ résistance thermique totale \\
\hline
\end{tabular}

Des calculs d'optimisation sous contraintes ont été effectués par L. Meysenc [7], [12] à l'aide d'algorithmes génétiques, et par C. Perret [13] avec le logiciel PASC OMA [14], qui utilise des algorithmes numériques 
d'optimisation. Les algorithmes génétiques sont particulièrement bien adaptés aux problèmes thermiques, puisqu'ils s'affranchissent assez bien des discontinuités (passage laminaire - turbulent). De plus, les deux types d'outils permettent de fixer le domaine d'évolution des grandeurs géométriques, tenant compte des critères de réalisation. Par exemple, la largeur du canal est fixée par la taille de la fraise scie utilisée pour l'usinage et ne peut être inférieure à une certaine valeur (de l'ordre de $200 \mu \mathrm{m}$ ).

\subsection{Validation du modèle et réalisations technologiques}

L. Meysenc a réalisé des prototypes monopuce en cuivre pour valider le modèle analytique donnant le coefficient d'échange en régime laminaire établi. Les prototypes étaient constitués d'une puce d'IGBT brasée sur un bloc de cuivre. Les microcanaux ont été usinés sur une surface égale à celle de la puce. La largeur des canaux était de l'ordre de quelques centaines de micromètres $(230$ et $311 \mu \mathrm{m})$. C. Perret a exploité les possibilités offertes par le développement des microsystèmes pour concevoir le même type de refroidisseur dans un support en silicium.

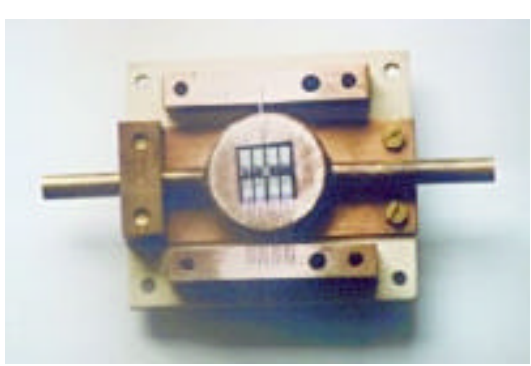

Figure 2: Photographie d'un prototype en cuivre [7]

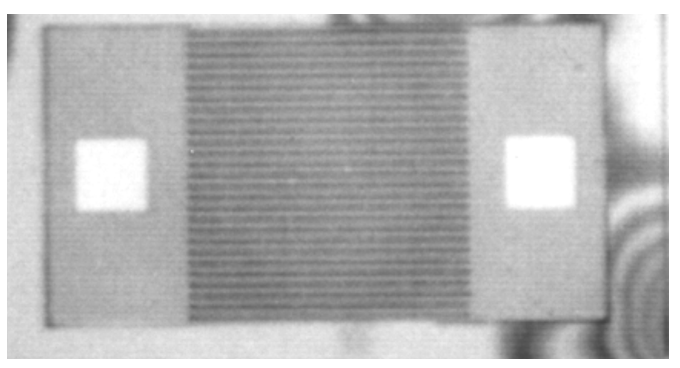

Figure 3 : Photographie d'un prototype en silicium [13]

C. Gillot a étendu cette démarche au module de puissance en développant un refroidisseur double face. Elle a réalisé des prototypes multipuces [8] présentés figure 4 et 5 .

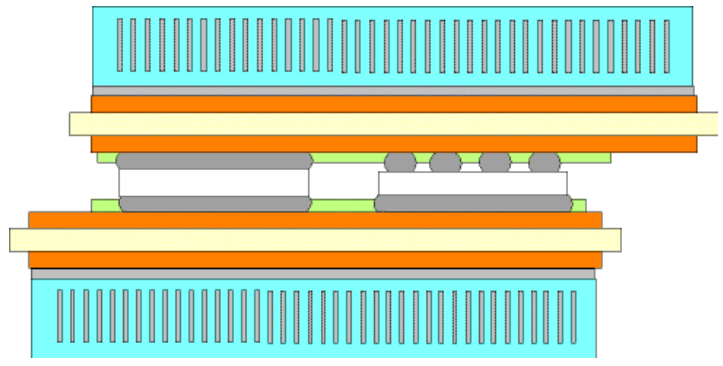

Figure 4: Principe du prototype double face [8]

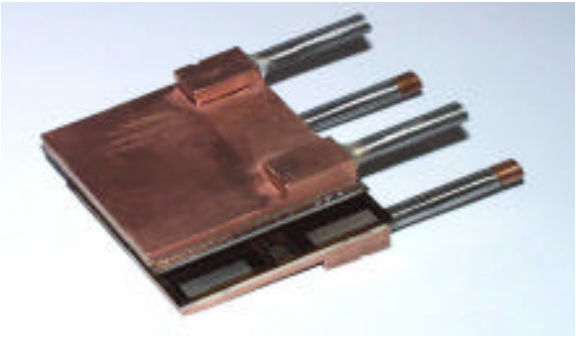

Figure 5 : Photographie d'un prototype double face [8]

\section{Fonctionnement d'un caloduc utilisé comme répartiteur de chaleur (spreader)}

Actuellement, la taille des composants électroniques a tendance à diminuer. Dans un même temps, leurs pertes thermiques augmentent considérablement. En conséquence, les densités de puissance à dissiper par les refroidisseurs deviennent très importantes. Afin de pallier ce problème, on utilise généralement des radiateurs plus grands que les composants afin d'épanouir au mieux le flux de chaleur. Mais cette solution mène généralement à l'apparition de points chauds. Le flux de chaleur n'est alors pas uniforme au niveau du radiateur. Celui ci n'est donc pas utilisé de façon optimale. En conséquence, la taille du radiateur à choisir est supérieure à celle que l'on prendrait si le flux de chaleur était uniforme. Afin de limiter ces points chauds, des matériaux à forte conductivité thermique tels que le diamant ou un de ses composites peuvent être utilisés. Leur prix n'étant pas très abordable, on essaie de les remplacer par des caloducs utilisés comme répartiteurs de chaleur. De tels produits sont actuellement industrialisés. Il s'agit, entre autre, de caloducs plats métalliques dont le réseau capillaire est constitué de poudres frittées. Côté recherche, des structures en silicium ont été présentées. Il s'agit de caloducs à rainures fines gravées dans le silicium [15]. L'avantage de ce matériau est qu'il pourrait, à terme, permettre de réaliser un spreader en face arrière du composant "sans sortir de la salle blanche". Cela représenterait en effet un avantage notable en terme de coût, de compacité et de crédibilité industrielle de la fonction. Afin de montrer l'intérêt de ce type de structure, nous allons présenter un exemple de spreader pouvant être réalisé en silicium. Nous verrons ensuite quelles sont les performances que l'on peut en attendre. 


\subsection{Principe de fonctionnement d'un spreader [1]}

Les phénomènes physiques permettant le fonctionnement d'un spreader sont les même que ceux intervenant dans les caloducs. La figure 6 décrit le principe de fonctionnement de cette structure.

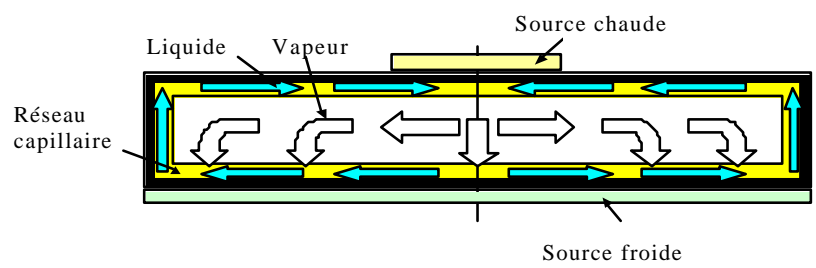

Figure 6 : Principe d'un spreader [1]

Comme pour un caloduc, au niveau de la source chaude, le liquide contenu dans le réseau capillaire s'évapore. La vapeur obtenue se déplace vers la source froide et s'y condense. Le retour du liquide à l'évaporateur s'effectue grâce au réseau capillaire se trouvant sur les parois internes. Nous avons choisi de réaliser un spreader dont le réseau capillaire est constitué de rainures axiales rectangulaires. En effet, d'une part leur gravure ne pose pas de problème sur un substrat silicium. D'autre part, Hopkins et coll. [16] ont montré qu'il est possible de faire transiter des puissances supérieures à 100 Watts sur une longueur efficace proche de $10 \mathrm{~cm}$ grâce à un réseau capillaire constitué de rainures rectangulaires de largeur $200 \mu \mathrm{m}$ (surface de chauffe proche de $1 \mathrm{~cm}^{2}$ ).

\subsection{Modèle thermohydraulique}

Si on veut prévoir la chute de température entre la source chaude et la source froide, il est nécessaire de connaître la conductivité de toutes les zones du caloduc. En particulier, une connaissance de la conductivité équivalente des rainures au niveau du condenseur et de l'évaporateur est nécessaire. Le modèle présenté ici est celui de Chi [17]. Il propose des expressions permettant de calculer la conductivité équivalente à l'évaporateur (7) et celle au condenseur (8).

$k_{e}=\frac{L_{1} k_{l} k_{w} t_{g}+k_{l} W\left(0.185 L_{1} k_{w}+t_{g} k_{l}\right)}{\left(W+L_{1}\right)\left(0.185 L_{1} k_{w}+t_{g} k_{l}\right)}(7) \quad$ et $\quad k_{c}=\frac{W k_{l}+L_{1} k_{w}}{W+L_{1}}$

Les limites de fonctionnement prédominantes des caloducs miniatures sont la limite capillaire et la limite d'ébullition. Afin de calculer la première, nous proposons une méthode permettant de connaître les pertes de pression liquide et une autre permettant de calculer les pertes de pression vapeur.

\subsubsection{Pertes de pression liquide}

Les hypothèses de calcul sont : le ménisque est attaché au haut de la rainure ; la section de liquide est supposée constante le long du caloduc; l'écoulement est en régime permanent, laminaire, incompressible et unidimensionnel; il n'y a pas d'interaction liquide-vapeur ; le réseau capillaire est saturé de liquide et le spreader est en position horizontale (pas d'action de la gravité).

Avec ces hypothèses, Chi [17] a montré dans le cas d'un caloduc classique, qu'il est possible de relier les pertes de pression au flux de chaleur axial local Q (9).

$$
\begin{aligned}
& \frac{d P_{l}}{d x}=-\frac{\left(f_{l} \operatorname{Re}_{l}\right) \mu_{l}}{2 \varepsilon A_{w} r_{h, l}^{2} h_{f g}} Q(9) \text { avec } f_{l} \operatorname{Re}_{l}=24\left(1-1.3553 c+1.9467 c^{2}-1.7012 c^{3}+0.9564 c^{4}-0.2537 c^{5}\right) \\
& \text { et } c=\max \left(W / t_{g}, t_{g} / W\right) \text { pour le cas des rainures axiales rectangulaires. }
\end{aligned}
$$

\subsubsection{Pertes de pression vapeur}

L'écoulement de vapeur dans un caloduc utilisé comme répartiteur de chaleur est difficile à modéliser à cause des dissymétries. En effet, dans les configurations axisymétriques, on admet que le profil de vitesse dans une section de conduit est parabolique ou turbulent avec une vitesse maximale au centre de ce conduit. Des calculs analytiques sont alors possibles. Dans le cas d'un spreader, le champ de vitesse n'a pas du tout cette allure. Une modélisation numérique 3D est donc nécessaire. Pour calculer le profil de pression vapeur, nous avons utilisé le logiciel Fluent. Pour cela, nous avons considéré que l'évaporation pouvait être modélisée comme une injection 
de vapeur de vitesse $Q_{t o t} / \rho_{v} h_{f g} L_{e} l$ perpendiculaire à l'évaporateur. De même la condensation a été considérée comme une sortie de vapeur de vitesse $Q_{t o t} / \rho_{v} h_{f g} L_{c} l$ perpendiculaire au condenseur.

\subsubsection{Limite capillaire}

Il est possible d'écrire un équilibre de pressions entre deux points $\mathrm{x}_{1}$ et $\mathrm{x}_{2}$ situés à l'interface liquide vapeur de la manière suivante :

$$
\left[P_{v}\left(x_{1}\right)-P_{v}\left(x_{2}\right)\right]+\left[P_{v}\left(x_{2}\right)-P_{l}\left(x_{2}\right)\right]+\left[P_{l}\left(x_{2}\right)-P_{l}\left(x_{1}\right)\right]+\left[P_{l}\left(x_{1}\right)-P_{v}\left(x_{1}\right)\right]=0
$$

Cette équation peut s'écrire d'une manière différente en faisant intervenir la pression capillaire, notée $\mathrm{P}_{\mathrm{c}}$, qui est la différence de pression entre la phase liquide et la phase gazeuse en un point de l'interface. Nous avons donc :

$$
P_{c}\left(x_{2}\right)=P_{c}\left(x_{1}\right)+\Delta P_{v}\left(x_{2}-x_{1}\right)+\Delta P_{l}\left(x_{1}-x_{2}\right)
$$

où $\Delta \mathrm{P}_{\mathrm{v}}$ et $\Delta \mathrm{P}_{1}$ sont les différences de pressions de la vapeur et du liquide dues à l'écoulement du fluide caloporteur. On choisit les points $x_{1}$ et $x_{2}$ comme étant respectivement le point mouillé (point où la pression capillaire est minimale) et le point sec (point où la pression capillaire est maximale). Si on fait l'hypothèse que $P c\left(x_{1}\right)$ est égale à zéro (angle de contact $\theta=90^{\circ}$ ), on peut alors réécrire l'équation précédente de la manière suivante :

$$
P_{c}\left(x_{2}\right)=\Delta P_{v}\left(x_{2}-x_{1}\right)+\Delta P_{l}\left(x_{1}-x_{2}\right)
$$

Or le réseau capillaire à rainure a une pression capillaire maximale Pcmax qui est égale à $2 \sigma \cos (\theta) / W$. Pour que le caloduc puisse fonctionner, il faudra que $\mathrm{Pc}\left(\mathrm{x}_{2}\right)$ reste toujours inférieure à cette valeur. La limite capillaire sera donc atteinte lorsque :

$$
\frac{2 \sigma \cos \theta)}{W}=\Delta P_{v}\left(x_{2}-x_{1}\right)+\Delta P_{l}\left(x_{1}-x_{2}\right)
$$

Comme il a été dit auparavant, le point mouillé est le point pour lequel la pression capillaire est nulle, donc pour le trouver, il suffit de faire tangenter les courbes des profils de pression liquide et vapeur. Ensuite, pour déterminer le point sec, on soustrait les deux profils tangents et le maximum se trouve être le point sec. La valeur de pression en ce point est la pression capillaire au point sec, c'est donc cette valeur qui ne doit pas dépasser la pression capillaire maximale. La limite capillaire de la structure étudiée a été calculée grâce à cette méthode (figure 7).

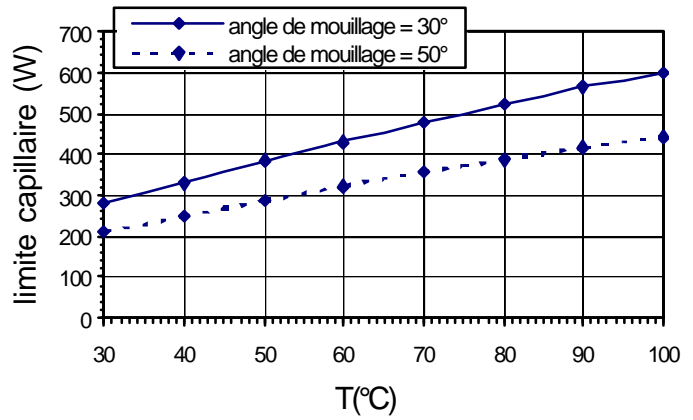

Figure 7 : Limite capillaire

On voit que pour les dimensions imposées, elle est très importante (supérieure à 200W). Cette valeur paraît logique car si on fait un calcul rapide de la longueur efficace liquide, on trouve une valeur proche du centimètre. Les chutes de pression liquide sont donc très faibles. En conséquence, la limite d'ébullition sera très certainement prépondérante à haute température. Le calcul de celle ci n'est pas présentée car cette limite dépend de beaucoup de paramètres expérimentaux (méthode de remplissage, matériau enveloppe, fluide ...)

\subsection{Réalisation d'un prototype}

Avec des procédés de gravure profonde réactive (DRIE) et de collage de plaques de silicium (WB) plusieurs prototypes ont été élaborés pour valider ces dimensionnements. Ces réalisations technologiques ont été effectuées par le CEA/LETI de Grenoble dans le département des Technologies Silicium. Les bancs de test ont été réalisés au LEG. 


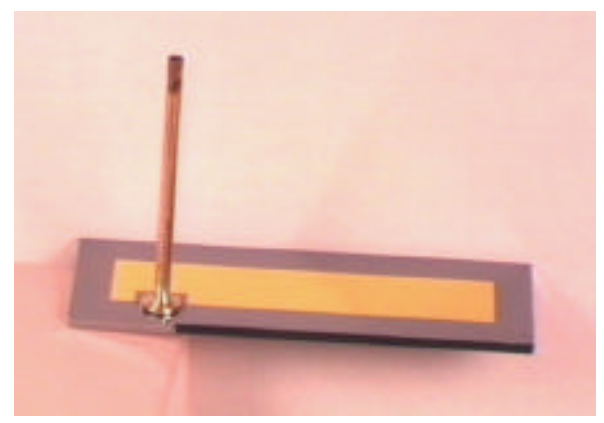

Figure 8: Prototype de spreader en silicium

\section{Conclusion}

L'utilisation des stratégies de microthermie pour refroidir les systèmes électriques paraît très intéressante. Les premiers produits industriels existent actuellement sur le marché. Dans cet article, nous avons essayé de montrer les potentialités qu'offraient les microrefroidisseurs à simple et double phase. Une réalisation de spreader à rainures axiales réalisé en silicium grâce aux techniques de la microélectronique a été présentée. Le modèle thermohydraulique et les expérimentations conduites nous permet tent de penser que les puissances maximales admissibles par ce type de structure peuvent être très importantes (supérieures à la centaine de Watt pour un surface de chauffage de $1 \mathrm{~cm}^{2}$ ).

\section{Bibliographie :}

[1] Y. Avenas, “ Etude et réalisation de caloducs plats miniatures pour l'intégration en électronique de puissance.", Thèse de doctorat de l'INPG, Grenoble, Décembre 2002.

[2] D.B. Tuckerman, R.F.W. Pease, "High-performance heat sink for VLSI", IEEE Electron Devices Letters, vol EDL-2, May 1981, pp. 126-129.

[3] R.J. Phillips, "Microchannel heat sinks", in Advances in Thermal Modeling of Electronic Components, New York: ASME, 1990, vol.2, ch. 3, pp. 109-184.

[4] R.K. Knight and Co, "Heat sink optimization with application to microchannels", IEEE Transactions on Components, Hybrids and Manufacturing Technology, vol. 15, Oct. 1992, pp. 832-842.

[5] A. Weisberg H.H. Bau, "Analysis of microchannels for integrated cooling”, International Journal of Heat and Mass Transfer, vol. 35, 1992, pp. 2465-2474.

[6] D. Copeland and Co, "Manifold microchannel heat sinks: isothermal analysis", IEEE Transactions on Components, Packaging, and Manufacturing Technology - Part A, vol. 20, June 1997, pp. 96-102.

[7] L. Meysenc, "Etude des micro-échangeurs intégrés pour le refroidissement des semíconducteurs de puissance”, Thèse de doctorat de l'INPG, Grenoble, Février 1998.

[8] C. Gillot, "Etude des micro-échangeurs intégrés pour le refroidissement des semiconducteurs de puissance", Thèse de doctorat de l'INPG, Grenoble, Février 1998.

[9] B.X. Wang, X.F. Peng, “Experimental investigation on liquid forced-convection heat transfer through microchannels", International Journal of Heat and Mass Transfer, vol. 37, 1994, pp. 73-82.

[10] A. Bejan, “Convection heat transfer", New York : Wiley, 1984, pp. 75-82.

[11] J. Taine, J.P. Petit, “Transferts thermiques et mécaniques des fluides anisothermes”, Edition Dunod, France, 1989.

[12] L. Meysenc, L. Saludjian, A. Bricard, S. Rael, C. Schaeffer, "A high heat flux IGBT micro exchanger setup”, IEEE Transactions on Components, Packaging, and Manufacturing Technology - Part A, vol. 20, Sept. 1997, pp. 334341.

[13] C. Perret, J. Boussey, C. Schaeffer, M. Coyaud, "Integration of cooling devices in silicon technology", Proceedings IAS'99, Phoenix, Arizona, October 3-7, 1999, pp. 1780-1786.

[14] F. Wurtz, J. Bigeon, "A methodology and a tool for the computer aided design with constraints of electrical devices”, IEEE Transaction on Magnetics, vol. 32, May 1996.

[15] D.A. Benson, R.T. Mitchell, M.R. Tuck, D.W. Palmer, G.P. Peterson, "Ultrahigh Capacity Micromachined Heat Spreaders", Microscale Thermophysical Engineering, Vol. 2, pp. 21-30, 1998.

[16] R. Hopkins, A. Faghri, D. Khrustalev, "Flat Miniatures Heat Pipes With Micro Capillary Grooves", Journal of Heat Transfer, Vol. 121, Feb. 1999, pp. 102-109.

[17] S.W. Chi, "Heat Pipe Theory and Practice", McGraw -Hill, 1976. 


\section{Nomenclature}

$A_{w} \quad$ surface réseau capillaire, $\mathrm{m}^{2}$

$C_{p} \quad$ chaleur spécifique, $\mathrm{J} \mathrm{kg}^{-1} \cdot \mathrm{K}^{-1}$

$D_{h} \quad$ diamètre hydraulique, $\mathrm{m}$

$f \quad$ coefficient de frottement

$h \quad$ coefficient d'échange, W. $\mathrm{m}^{-2} \cdot \mathrm{K}^{-1}$

$h_{f g} \quad$ chaleur latente d'évaporation, ${\mathrm{J} . \mathrm{kg}^{-1}}^{-1}$

$K$ perméabilité, $\mathrm{m}^{2}$

$k$ conductivité thermique, W. $\mathrm{K}^{-1} \cdot \mathrm{m}^{-1}$

$l \quad$ largeur de l'espace vapeur, $\mathrm{m}$

$L \quad$ longueur, $\mathrm{m}$

$L_{1} \quad$ largeur ailette, $\mathrm{m}$

$n$ nombre d'ailettes

$\mathrm{Nu}$ nombre de Nusselt

$P$ pression, $\mathrm{Pa}$

$P r \quad$ nombre de Prandtl

$Q \quad$ flux de chaleur axial local, W

$Q_{\text {tot }} \quad$ flux de chaleur total, W

Re nombre de Reynolds

$r_{h} \quad$ rayon hydraulique, $\mathrm{m}$

$R_{t h} \quad$ résistance thermique, $\mathrm{K}$

$T$ température, $\mathrm{K}$

$t$ épaisseur paroi, $\mathrm{m}$

$t_{g} \quad$ profondeur des rainures, $\mathrm{m}$

$t_{v} \quad$ hauteur espace vapeur, $\mathrm{m}$

$W$ largeur rainure

Symboles grecs

$\beta \quad$ efficacité d'ailette

$\varepsilon \quad$ porosité

$\mu \quad$ viscosité dynamique, $\mathrm{Pa} . \mathrm{s}^{-1}$

$\theta \quad$ angle de contact

$\rho \quad$ masse volumique, $\mathrm{kg} \cdot \mathrm{m}^{-3}$

$\sigma \quad$ tension surfacique, $\mathrm{N} . \mathrm{m}^{-1}$

$\tau \quad$ contrainte pariétale

Indices et exposants

$c$ condenseur

$e \quad$ évaporateur

$l \quad$ liquide

$v \quad$ vapeur 